\title{
SVD-Krylov based Sparsity-preserving Techniques for Riccati-based Feedback Stabilization of Unstable Power System Models
}

\author{
Mahtab Uddin ${ }^{*}, 1,3$, M. Monir Uddin ${ }^{2}$, M. A. Hakim Khan ${ }^{3}$, M. Tanzim Hossain ${ }^{4}$ \\ ${ }^{1}$ Institute of Natural Sciences, United International University, Dhaka-1212, Bangladesh \\ ${ }^{2}$ Department of Mathematics and Physics, North South University, Dhaka-1229, Bangladesh \\ ${ }^{3}$ Department of Mathematics, Bangladesh University of Engineering \& Technology, Dhaka-1000, Bangladesh \\ ${ }^{4}$ Department of Electrical and Computer Engineering, North South University, Dhaka-1229, Bangladesh
}

Received: May 01, 2021, Revised: July 20, 2021, Accepted: July 24, 2021, Available Online: August 20, 2021

\begin{abstract}
We propose an efficient sparsity-preserving reduced-order modelling approach for index-1 descriptor systems extracted from largescale power system models through two-sided projection techniques. The projectors are configured by utilizing Gramian based singular value decomposition (SVD) and Krylov subspace-based reduced-order modelling. The left projector is attained from the observability Gramian of the system by the low-rank alternating direction implicit (LR-ADI) technique and the right projector is attained by the iterative rational Krylov algorithm (IRKA). The classical LR-ADI technique is not suitable for solving Riccati equations and it demands high computation time for convergence. Besides, in most of the cases, reduced-order models achieved by the basic IRKA are not stable and the Riccati equations connected to them have no finite solution. Moreover, the conventional LR-ADI and IRKA approach not preserves the sparse form of the index-1 descriptor systems, which is an essential requirement for feasible simulations. To overcome those drawbacks, the fitting of LR-ADI and IRKA based projectors from left and right sides, respectively, desired reduced-order systems attained. So that, finite solution of low-rank Riccati equations, and corresponding feedback matrix can be executed. Using the mechanism of inverse projection, the Riccati-based optimal feedback matrix can be computed to stabilize the unstable power system models. The proposed approach will maintain minimized $\mathfrak{H}_{2}$-norm of the error system for reduced-order models of the target models.
\end{abstract}

Keywords: Singular Value Decomposition, Krylov Subspace, Alternative Direction Implicit, Riccati Equation, $\mathcal{H}_{2}$-norm, Optimal Feedback Stabilization.

This work is licensed under a Creative Commons Attribution-Non Commercial 4.0 International License.

\section{Introduction}

The index-1 descriptor systems of the first-order are conventionally the technical arrangement of sparse sub-matrices with appropriate structure. These sub-matrices are arranged in system-oriented input-output combinations. These systems can be formed as

$$
\begin{gathered}
E_{11} \dot{x_{1}}(t)=A_{11} x_{1}(t)+A_{12} x_{2}(t)+B_{1} u(t), \\
0=A_{21} x_{1}(t)+A_{22} x_{2}(t)+B_{2} u(t), \\
y(t)=C_{1} x_{1}(t)+C_{2} x_{2}(t)+D u(t), \\
x\left(t_{0}\right)=x_{0}, \quad t \geq t_{0} .
\end{gathered}
$$

In system (1), $E_{11} \in \mathbb{R}^{\mathrm{n}_{1} \times \mathrm{n}_{1}}$ is the differential coefficient matrix, and $A_{11} \in \mathbb{R}^{\mathrm{n}_{1} \times \mathrm{n}_{1}}, \quad A_{12} \in \mathbb{R}^{\mathrm{n}_{1} \times \mathrm{n}_{2}}, \quad A_{21} \in \mathbb{R}^{\mathrm{n}_{2} \times \mathrm{n}_{1}}$, $A_{22} \in \mathbb{R}^{\mathrm{n}_{2} \times \mathrm{n}_{2}}$ are the state sub-matrices. The control multiplier sub-matrices $B_{1} \in \mathbb{R}^{\mathrm{n}_{1} \times \mathrm{p}}, B_{2} \in \mathbb{R}^{\mathrm{n}_{2} \times \mathrm{p}}$ with the state multiplier sub-matrices $C_{1} \in \mathbb{R}^{\mathrm{m} \times \mathrm{n}_{1}}, C_{2} \in \mathbb{R}^{\mathrm{m} \times \mathrm{n}_{2}}$. The direct gain matrix $D \in \mathbb{R}^{\mathrm{m} \times \mathrm{p}}$ is for the input to output transfer without alteration, it remains zero in many of the physical systems, for instance, power systems models. Here, the system dimension of the system (1) is $n=n_{1}+n_{2}$ with the dimensions of input and output $p$ and $m$, respectively. In the case of large-scale systems $n$ is very large, whereas $p, m$ are comparatively smaller. The vectors $x_{1}(t) \in \mathbb{R}^{\mathrm{n}_{1}}, x_{2}(t) \in \mathbb{R}^{\mathrm{n}_{2}}$ are for state vectors, whereas $u(t) \in \mathbb{R}^{p}$ and $y(t) \in \mathbb{R}^{m}$ represent the input (control) and output vectors, respectively. The sub-matrices $E_{11}$ and $A_{22}$ have the full rank [1]-[2].
For further manipulation, $x_{2}(t)=-A_{22}^{-1} A_{21} x_{1}(t)-$ $A_{22}^{-1} B_{2} u(t)$ needs to be eliminated from the algebraic (second) part of the Equation (1). Then the Schur complements of the system (1) can be formed as

$$
\begin{gathered}
x:=x_{1}, \quad \mathcal{E}:=E_{11}, \quad \mathcal{A}:=A_{11}-A_{12} A_{22}^{-1} A_{21}, \\
\mathcal{B}:=B_{1}-A_{12} A_{22}^{-1} B_{2}, \mathcal{C}:=C_{1}-C_{2} A_{22}^{-1} A_{21}, \\
\mathcal{D}:=D-C_{2} A_{22}^{-1} B_{2} .
\end{gathered}
$$

Using the Schur complements given in (2), the index-1 descriptor system (1) can structure into an analogous generalized LTI continuous-time system as

$$
\begin{array}{r}
\mathcal{E} \dot{x}(t)=\mathcal{A} x(t)+\mathcal{B} u(t), \\
y(t)=\mathcal{C} x(t)+\mathcal{D} u(t) .
\end{array}
$$

Applying Laplace transformation, the transfer function for the system (3) can be found as

$$
G(s)=\mathcal{C}(s \mathcal{E}-\mathcal{A})^{-1} \mathcal{B}+\mathcal{D} ; \quad s \in \mathbb{C} .
$$

The implications of LTI continuous-time systems are inescapable in the branches of engineering fields with the applications of applied mathematics, for example, system and control theory, mechatronics, power electronics [3]-[5]. Continuous-time Algebraic Riccati Equation (CARE) plays a premier role in engineering applications, such as the systems that 
originated from mechanical and electrical fields [6]-[7] . The CARE yields from the system (3) can be formed as

$$
\mathcal{A}^{T} X \mathcal{E}+\mathcal{E}^{T} X \mathcal{A}-\mathcal{E}^{T} X \mathcal{B} \mathcal{B}^{T} X \mathcal{E}+\mathcal{C}^{T} \mathcal{C}=0 .
$$

If all of the eigenvalues of the Hamiltonian matrix of the system (3) lie outside the imaginary axis, the solution $X$ of the CARE (5) is then unique and finite [8]. The symmetric positive-definite Matrix $X$ is called stabilizing if the closedloop matrix $\mathcal{A}-\left(\mathcal{B B}^{T}\right) X \mathcal{E}$ exists and is stable. Some of the systems, that have eigenvalues very close to the imaginary axis are called semi-stable systems. For an unstable type of system (3), the optimal feedback matrix $K^{o}=\mathcal{B}^{T} X \mathcal{E}$ needs to estimate to apply the Riccati-based feedback stabilization [9]. Implementing the desired $K^{o}$, the matrix $\mathcal{A}_{s}=\mathcal{A}-B K^{o}$ can be formed to replace the system matrix $\mathcal{A}$. Then the optimally stabilized target system can be written as

$$
\begin{aligned}
\mathcal{E} \dot{x}(t) & =\mathcal{A}_{s} x(t)+\mathcal{B} u(t), \\
y(t) & =\mathcal{C} x(t)+\mathcal{D} u(t) .
\end{aligned}
$$

In the previous works, we have discussed the rational Krylov subspace method (RKSM) and the Kleinman-Newton technique for the Riccati-based feedback stabilization technique for unstable index-1 descriptor systems [10]-[11]. The linear quadratic regulator (LQR) approach is the key of the RKSM and the low-rank Cholesky-factor integrated alternative direction implicit (LRCF-ADI) approach requires in the Kleinman-Newton technique. A modified form of the iterative rational Krylov algorithm (IRKA) technique was recently derived to treat that of the systems through optimal feedback stabilization [12]. In those works, some unstable systems extracted from the Brazilian Interconnected Power System (BIPS) models are deliberated to stabilize.

In this work, we introduce two-sided projection techniques for Riccati-based feedback stabilization for unstable BIPS models utilizing reduced-order modeling, which is a coupled approach of singular value decomposition and Krylov subspace and naming as Iterative SVD-Krylov Algorithm (ISKA). The reduced-order models will be validated through the $\mathcal{H}_{2}$ norm of the error system. Comparative discussion on the present work and IRKA approaches will be done.

\section{Preliminaries}

The Lyapunov equation consisting of observability Gramian $\mathcal{Q}$ of the system (3) has the form

$$
\mathcal{A}^{T} \mathcal{Q} \mathcal{E}+\mathcal{E}^{T} \mathcal{Q} \mathcal{A}+\mathcal{C}^{T} \mathcal{C}=0 .
$$

Computation of the $\mathcal{Q}$ by solving the Equation (7) containing large-scale matrices by the direct solvers is infeasible for the large-scale systems, sometimes it may impossible for the rising size of the system components. Thus, the observability Gramian factor $Z_{q}$ needs to be estimated by any feasible approach. There are some efficient techniques available for executing $Z_{q}$, for example, low-rank Choleskyfactor-based Alternating Direction Implicit (LRCF-ADI) [13][14]. Then the Gramian, $\mathcal{Q}=Z_{q} Z_{q}^{T}$ can be approximated as the solution of the Lyapunov Equation (7). The LRCF-ADI approach for computing $Z_{q}$ is provided in Algorithm 1 .
Algorithm 1: First-order LRCF-ADI Algorithm [13]

Input: $\quad \mathcal{E}, \mathcal{A}, \mathcal{B}, \mathcal{C}, \tau$ (tolerance), $i_{\max }$ (iterations), and $\left\{\mu_{j}\right\}_{j=1}^{i_{\max }}$ (initial shifts).

Output: $\quad$ Low-rank Cholesky-factor $Z_{q}$ for $\mathcal{Q}=Z_{q} Z_{q}^{T}$.

1 Assume at $i=1, Z_{0}=[]$ and $W_{0}=C^{T}$.

2 while $\left\|\mathcal{W}_{i-1} \mathcal{W}_{i-1}^{T}\right\| \geq \tau$ or $i \leq i_{\max }$ do

$3 \quad$ Solve $\mathcal{V}_{i}=\left(\mathcal{A}^{T}+\mu_{i} \mathcal{E}^{T}\right)^{-1} \mathcal{W}_{i-1}$.

$4 \quad$ if $\operatorname{Im}\left(\mu_{i}\right)=0$ then

5

7

8

9

$$
\mid \begin{aligned}
& \text { Update } Z_{i}=\left[\begin{array}{ll}
Z_{i-1} & \sqrt{-2 \mu_{i}} \mathcal{V}_{i}
\end{array}\right] \\
& \text { Compute } \mathcal{W}_{i}=\mathcal{W}_{i-1}-2 \mu_{i} \mathcal{E}^{T} \mathcal{V}_{i}
\end{aligned}
$$

else

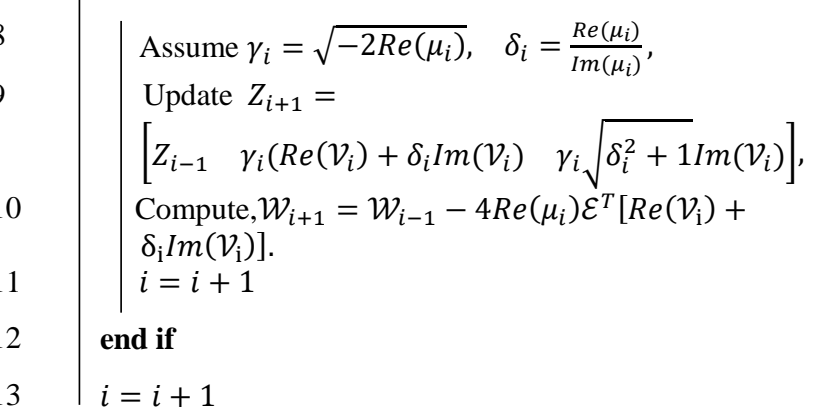

4 end while

Considering the computationally feasible $r$-dimensional reduced-order model (ROM) of the system (3) as

$$
\begin{aligned}
\hat{\mathcal{E}} \dot{\hat{x}}(t) & =\hat{\mathcal{A}} \hat{x}(t)+\widehat{\mathcal{B}} \hat{u}(t), \\
\hat{y}(t) & =\hat{\mathcal{C}} \hat{x}(t)+\widehat{\mathcal{D}} \hat{u}(t),
\end{aligned}
$$

where $\hat{\mathcal{E}} \in \mathbb{R}^{r \times r}, \hat{\mathcal{A}} \in \mathbb{R}^{r \times r}, \widehat{\mathcal{B}} \in \mathbb{R}^{r \times p}, \hat{\mathcal{C}} \in \mathbb{R}^{m \times r}$ and $\widehat{\mathcal{D}} \in$ $\mathbb{R}^{m \times p}$.

The reduced coefficient matrices of (8) are formed by the following way

$$
\begin{gathered}
\hat{\mathcal{E}}=W^{T} \mathcal{E} V, \quad \hat{\mathcal{A}}=W^{T} \mathcal{A} V, \quad \widehat{\mathcal{B}}=W^{T} \mathcal{B}, \\
\hat{\mathcal{C}}=\mathcal{C} V, \quad \widehat{\mathcal{D}}=\mathcal{D} .
\end{gathered}
$$

The transfer function of the ROM (8) can be found as

$$
\widehat{G}(s)=\hat{\mathcal{C}}(s \hat{\mathcal{E}}-\hat{\mathcal{A}})^{-1} \widehat{\mathcal{B}}+\widehat{\mathcal{D}} ; \quad s \in \mathbb{C} .
$$

The right projector $V$ is built by the well-known Krylovbased interpolatory techniques IRKA given in [15]-[16] as

$$
V=\left[\left(\alpha_{1} \mathcal{E}-\mathcal{A}\right)^{-1} \mathcal{B} b_{1}, \ldots,\left(\alpha_{r} \mathcal{E}-\mathcal{A}\right)^{-1} \mathcal{B} b_{r}\right],
$$

where $\left\{\alpha_{i}\right\}_{i=1}^{r}$ and $\left\{b_{i}\right\}_{i=1}^{r}$ are the interpolation points and tangential direction respectively. The left projector $W$ is computed by the observability Gramian $\mathcal{Q}$ utilizing the singular value decomposition-based techniques discussed in [17]-[19] as

$$
W=\mathcal{Q} V\left(V^{T} \mathcal{Q} V\right)^{-1} \text {. }
$$

The successive steps of the computation of the ROM (8) are exhibited in Algorithm 2. 
Algorithm 2: First-order ISKA [17]

Input: $\quad \mathcal{E}, \mathcal{A}, \mathcal{B}, \mathcal{C}, \mathcal{D}$ and $Z_{q}$ (from Algorithm 1 ).

Output: $\quad \hat{\mathcal{E}}, \hat{\mathcal{A}}, \widehat{\mathcal{B}}, \hat{\mathcal{C}}, \widehat{\mathcal{D}}:=\mathcal{D}$.

1 Choose the initial interpolation points $\left\{\alpha_{i}\right\}_{i=1}^{r}$ and the tangential directions $\left\{b_{i}\right\}_{i=1}^{r}$.

2 Construct $V=\left[\left(\alpha_{1} \mathcal{E}-\mathcal{A}\right)^{-1} \mathcal{B} b_{1}, \ldots,\left(\alpha_{r} \mathcal{E}-\mathcal{A}\right)^{-1} \mathcal{B} b_{r}\right]$.

3 Compute $\mathcal{Q}=Z_{q} Z_{q}^{T}$ and construct $W=\mathcal{Q} V\left(V^{T} \mathcal{Q} V\right)^{-1}$.

4 while (not converged) do

$5 \quad$ Find $\hat{\mathcal{E}}=W^{T} \mathcal{E} V, \hat{\mathcal{A}}=W^{T} \mathcal{A} V, \widehat{\mathcal{B}}=W^{T} \mathcal{B}, \hat{\mathcal{C}}=\mathcal{C} V$.

$6 \quad$ for $i=1, \ldots, r$. do

7

9

10

11

12 Repeat Step-5 to find the reduced-order matrices.

\section{Sparsity-preserving SVD-Krylov techniques for the stabilization of first-order index-1 descriptor system}

The objective of this work is to reduce the dimension of the first-order index-1 system (1) by keeping the sparse structure invariant through the Iterative SVD-Krylov Algorithm (ISKA) approach. To do this, it is essential to modify some steps of first-order ISKA and LRCF-ADI algorithms in terms of sparse sub-matrices.

3.1 Sparsity-preserving LRCF-ADI approach for the firstorder index-1 descriptor system

The LRCF-ADI method of first-order was discussed in [13],[20]-[21]. The modification of the LRCF-ADI algorithm for the structure-preserving second-order form can be derived as follows.

For the truncated term $\Gamma$, the first iteration of the Step-3 of Algorithm 1 can be written as

$$
\begin{gathered}
\left(\mathcal{A}^{T}+\mu_{1} \mathcal{E}^{T}\right) \mathcal{V}_{1}=\mathcal{C}^{T}, \\
\text { or, }\left(\left[\begin{array}{ll}
A_{11} & A_{12} \\
A_{21} & A_{22}
\end{array}\right]^{T}+\mu_{1}\left[\begin{array}{cc}
E_{11} & 0 \\
0 & 0
\end{array}\right]^{T}\right)\left[\begin{array}{l}
\mathcal{V}_{1} \\
\Gamma
\end{array}\right]=\left[\begin{array}{l}
C_{1}^{T} \\
C_{2}^{T}
\end{array}\right] .
\end{gathered}
$$

Thus, we have

$$
\left[\begin{array}{cc}
A_{11}^{T}+\mu_{1} E_{11}^{T} & A_{21}^{T} \\
A_{12}^{T} & A_{22}^{T}
\end{array}\right]\left[\begin{array}{l}
\mathcal{V}_{1} \\
\Gamma
\end{array}\right]=\left[\begin{array}{c}
C_{1}^{T} \\
C_{2}^{T}
\end{array}\right]
$$

As a consequence, for $i \geq 2$, the next $i-t h$ iteration takes the form

$$
\left[\begin{array}{cc}
A_{11}^{T}+\mu_{i} E_{11}^{T} & A_{21}^{T} \\
A_{12}^{T} & A_{22}^{T}
\end{array}\right]\left[\begin{array}{c}
\mathcal{V}_{i} \\
\Gamma
\end{array}\right]=\left[\begin{array}{c}
\mathcal{W}_{i-1} \\
0
\end{array}\right]
$$

If the estimated shift parameter has no imaginary part, then the Step- 6 of Algorithm 1 can be formed as

$$
\mathcal{W}_{i}=\mathcal{W}_{i-1}-2 \mu_{i} E_{11}^{T} \mathcal{V}_{i}
$$

Otherwise, for $\delta_{i}=\frac{\operatorname{Re}\left(\mu_{i}\right)}{\operatorname{Im}\left(\mu_{i}\right)}$, the Step-10 of Algorithm 1 can be expressed as

$$
\mathcal{W}_{i+1}=\mathcal{W}_{i-1}-4 \operatorname{Re}\left(\mu_{i}\right) E_{11}^{T}\left[\operatorname{Re}\left(\mathcal{V}_{i}\right)+\delta_{i} \operatorname{Im}\left(\mathcal{V}_{i}\right)\right]
$$

The restructured sparse form of LRCF-ADI for the firstorder index-1 system is exhibited in Algorithm 3.

Algorithm 3: LRCF-ADI for first-order sparse index-1 descriptor system

Input: $\quad E_{11}, A_{11}, A_{12}, A_{21}, A_{22}, B_{1}, B_{2}, C_{1}, C_{2}, \tau$

(tolerance), $i_{\text {max }}$ (iterations), and $\left\{\mu_{j}\right\}_{j=1}^{i_{\text {max }}}$ (initial shifts).

Output: Low-rank Cholesky-factor $Z_{q}$ for $\mathcal{Q} \approx Z_{q} Z_{q}^{T}$.

1 Assume at $i=1, Z_{0}=[]$ and $\mathcal{W}_{0}=\left[\begin{array}{ll}C_{1} & C_{2}\end{array}\right]^{T}$.

2 while $\left\|\mathcal{W}_{i-1} \mathcal{W}_{i-1}^{T}\right\| \geq \tau$ or $i \leq i_{\max }$ do

$3 \quad$ Solve (14) to find $\mathcal{V}_{1}$ and (15) to find $\mathcal{V}_{i} ; i \geq 2$.

$4 \quad$ if $\operatorname{Im}\left(\mu_{i}\right)=0$ then

5

6

Update $Z_{i}=\left[\begin{array}{ll}Z_{i-1} & \sqrt{-2 \mu_{i}} \\ \nu_{i}\end{array}\right]$,

Compute the updated value of $\mathcal{W}_{i}$ by (16)

7

8

else

10

11

12

end if

$13 \quad i=i+1$

14 end while

3.2 Sparsity-preserving ISKA for first-order index-1 descriptor system

Algorithm 2 needs to reform in the sparse form with the system matrices of (1). In the Step-2 of this algorithm, projector $V$ needs to be re-structured utilizing the first-order sparse matrices. Let us consider the $i-t h$ iteration of $V$ be expressed as $V_{i}$ and it can be configured as

$$
\begin{gathered}
\left(\alpha_{i} \mathcal{E}-\mathcal{A}\right) V_{i}=\mathcal{B} b_{i} \\
\text { or, }\left[\begin{array}{cc}
\alpha_{i} E_{11}-A_{11} & -A_{12} \\
-A_{21} & -A_{22}
\end{array}\right]\left[\begin{array}{c}
V_{i} \\
\Lambda
\end{array}\right]=\left[\begin{array}{c}
B_{1} \\
B_{2}
\end{array}\right] b_{i}
\end{gathered}
$$

The term $\Lambda$ is to be truncated. The explicit execution of the reduced-order matrices for the system (8) defined in (9) is infeasible and contradicts the aim of the work.

The reduced-order matrices can be efficiently acquired by the sparsity preserving form as

$$
\begin{gathered}
\hat{\mathcal{E}}=W^{T} E_{11} V, \quad \hat{A}=W^{T} A_{11} V-\left(W^{T} A_{12}\right) A_{22}^{-1}\left(A_{21} V\right), \\
\hat{B}=W^{T} B_{1}-\left(W^{T} A_{12}\right) A_{22}^{-1} B_{2} \\
\hat{C}=C_{1} V-C_{2} A_{22}^{-1}\left(A_{21} V\right) .
\end{gathered}
$$

The sparsity-preserving modified form of Algorithm 2 for the first-order index-1 system is summarized in Algorithm 4. 
Algorithm 4: ISKA for first-order sparse index-1 descriptor system

Input: $\quad E_{11}, A_{11}, A_{12}, A_{21}, A_{22}, B_{1}, B_{2}, C_{1}, C_{2}, D$ and $Z_{q}$ (from Algorithm 3 ).

Output: $\quad \hat{\mathcal{E}}, \hat{\mathcal{A}}, \widehat{\mathcal{B}}, \hat{\mathcal{C}}, \widehat{\mathcal{D}}:=D-C_{2} A_{22}^{-1} B_{2}$

1 Choose the initial interpolation points $\left\{\alpha_{i}\right\}_{i=1}^{r}$ and the tangential directions $\left\{b_{i}\right\}_{i=1}^{r}$.

2 Construct $V=\left[V_{1}, V_{2}, \ldots, V_{r}\right]$ using (18).

3 Compute $\mathcal{Q}=Z_{q} Z_{q}^{T}$ and construct $W=Q V\left(V^{T} Q V\right)^{-1}$.

4 while (not converged) do

$5 \quad$ Find the reduced-order matrices by (19).

6 for $i=1, \ldots, r$. do

$7 \quad$ Evaluate $\hat{\mathcal{A}} z_{i}=\lambda_{i} \hat{\mathcal{E}} z_{i}$ and $y_{i}^{*} \hat{\mathcal{A}}=\lambda_{i} y_{i}^{*} \hat{\mathcal{E}}$ to find $\alpha_{i} \leftarrow-\lambda_{i}, b_{i}^{*} \leftarrow-y_{i}^{*} \hat{B}$. end for

Repeat Step-2 and Step-3.

$10 \quad i=i+1$

11 end while

12 Repeat Step-5 to find the reduced-order matrices.

\subsection{Computing the optimal feedback matrix from ROM}

System (3) can be written in a reduced-order form as (8) by exerting the reduced-order matrices defined in (19) and corresponding CARE can be attained as

$$
\hat{\mathcal{A}}^{T} \hat{X} \hat{\mathcal{E}}+\hat{\mathcal{E}} \hat{X} \hat{\mathcal{A}}-\hat{\mathcal{E}} \hat{X} \widehat{\mathcal{B}} \widehat{\mathcal{B}}^{T} \hat{X} \hat{\mathcal{E}}+\hat{\mathcal{C}}^{T} \hat{\mathcal{C}}=0 .
$$

Here $\hat{X}$ has the same properties of $X$. The MATLAB library command care can be applied to solve the low-rank CARE (20). The low-rank stabilizing feedback matrix $\widehat{K}=$ $\widehat{B}^{T} \hat{X} \hat{\mathcal{E}}$ corresponding to the ROM (8) can be computed, and consequently approximated the stabilizing optimal feedback matrix $K^{o}$ of the full model (3) can be reclaimed employing the scheme of reverse projection as

$$
K^{o}=\left(\widehat{\mathcal{B}}^{T} \hat{X} \hat{\mathcal{E}}\right) V^{T} E_{11}=\widehat{K} V^{T} E_{11} .
$$

3.4 Optimally stabilized first-order index-1 descriptor system

For the original system (1), optimal feedback matrix $K^{o}$ can be attained by assigning the ROM (8). Then by utilizing $K^{o}$, the optimally stabilized system (1) can be found by replacing $A_{11}$ and $A_{21}$ by $A_{11}-B_{1} K^{o}$ and $A_{21}-B_{2} K^{o}$, respectively

\section{$3.5 \mathcal{H}_{\mathbf{2}}$-norm of the error system}

Now, the error system associated with the ROM (8) of the subjected system (1) by maintaining the form (3) has the form

$$
G_{\text {err }}=G(s)-\hat{G}(s)=\mathcal{C}_{\text {err }}\left(s \mathcal{E}_{\text {err }}-\mathcal{A}_{\text {err }}\right)^{-1} B_{\text {err }},
$$

where the transfer functions $G(s)$ and $\hat{G}(s)$ are connected to the systems (1) and (8), respectively. In (22), we have constituted

$$
\begin{aligned}
& \mathcal{E}_{\text {err }}=\left[\begin{array}{ll}
\mathcal{E} & 0 \\
0 & \hat{\mathcal{E}}
\end{array}\right], \quad \mathcal{A}_{\text {err }}=\left[\begin{array}{cc}
\mathcal{A} & 0 \\
0 & \hat{\mathcal{A}}
\end{array}\right], \\
& \mathcal{B}_{\text {err }}=\left[\begin{array}{l}
\mathcal{B} \\
\widehat{\mathcal{B}}
\end{array}\right] \text { and } \quad \mathcal{C}_{\text {err }}=\left[\begin{array}{ll}
\mathcal{C} & -\hat{\mathcal{C}}
\end{array}\right] .
\end{aligned}
$$

The observability Lyapunov equation corresponding to the Graminan $\mathcal{Q}_{\text {err }}$ of the error system (22) is

$$
\mathcal{A}_{\text {err }}^{T} \mathcal{Q}_{\text {err }} \mathcal{E}_{\text {err }}+\mathcal{E}_{\text {err }}^{T} \mathcal{Q}_{\text {err }} \mathcal{A}_{\text {err }}+\mathcal{C}_{\text {err }}^{T} \mathcal{C}_{\text {err }}=0
$$

For the error system (22), the Authors in [5] explored an efficient approach to approximate the $\mathcal{H}_{2}$-norm as

$$
=\frac{\left\|G_{\text {err }}\right\|_{\mathcal{H}_{2}}=\sqrt{\operatorname{trace}\left(\mathcal{B}_{\text {err }}^{T} \mathcal{Q}_{\text {err }} \mathcal{B}_{\text {err }}\right)}}{\|G(s)\|_{\mathcal{H}_{2}}^{2}+\|\hat{G}(s)\|_{\mathcal{H}_{2}}^{2}+2 \operatorname{trace}\left(\mathcal{B}^{T} \mathcal{Q}_{s} \widehat{\mathcal{B}}\right)}
$$

Here, $\|G(s)\|_{\mathcal{H}_{2}}$ is the $\mathcal{H}_{2}$-norm of the full model which we need to evaluate at one time in computation but this is unfeasible to investigate for a large-scale system by any direct solver. Suppose $Z_{q}$ is the low-rank Gramian factor that can be successfully determined by rearranging Algorithm 3, such that $Q=Z_{q} Z_{q}^{T}$, then $\|G(s)\|_{\mathcal{H}_{2}}$ can be written as

$$
\begin{gathered}
\|G(s)\|_{\mathcal{H}_{2}}^{2}=\operatorname{trace}\left(\mathcal{B}^{T} Q \mathcal{B}\right) \\
=\operatorname{trace}\left(B_{1}^{T}\left(Z_{q} Z_{q}^{T}\right) B_{1}+B_{2}^{T}\left(Z_{q} Z_{q}^{T}\right) B_{2}\right) .
\end{gathered}
$$

Again, the $\mathcal{H}_{2}$-norm of the $\operatorname{ROM},\|\widehat{G}(s)\|_{\mathcal{H}_{2}}$ can be enumerated by the Gramian $\hat{\mathcal{Q}}$ of the low-rank Lyapunov equation

$$
\hat{\mathcal{A}}^{T} \hat{\mathcal{Q}} \hat{\mathcal{E}}+\hat{\mathcal{E}}^{T} \hat{\mathcal{Q}} \hat{\mathcal{A}}+\hat{\mathcal{C}}^{T} \hat{\mathcal{C}}=0,
$$

that consists of reduced-order matrices. Due to the small size of these matrices, the following Lyapunov equation is solvable by the MATLAB library command lyap.

Finally, trace $\left(\mathcal{B}^{T} \mathcal{Q}_{s} \widehat{\mathcal{B}}\right)$ can be measured by the low-rank Gramian $\mathcal{Q}_{s}$ of the sparse-dense Sylvester equation

$$
\mathcal{A}^{T} \mathcal{Q}_{s} \hat{\mathcal{E}}+\mathcal{E}^{T} \mathcal{Q}_{S} \hat{\mathcal{A}}+\mathcal{C}^{T} \hat{\mathcal{C}}=0,
$$

that can be efficiently solved by the techniques presented in Algorithm 4 of [22].

\section{Numerical results}

The derived method ISKA is validated by implementing some models evolved from Brazilian Interconnected Power System (BIPS) [23]. The computations are performed numerically by MATLAB $^{\circledR}$ R2015a (8.5.0.197613) with a processor $4 \times$ Intel $^{\circledR}$ Core $^{\mathrm{TM}} \mathrm{i} 5-6200 \mathrm{U}$ incorporating a memory capacity of $16 \mathrm{~GB}$ with a clock speed of $2.30 \mathrm{GHz}$.

Table 1 displays the dimensions of the discussing models along with analogous input-output structures, and the size of the corresponding ROMs gained by the developed technique ISKA as illustrated in Algorithm 4.

Table 1 Model examples with input-output structures

\begin{tabular}{cccc}
\hline Model & Full model (n) & Input/Output & ROM (r) \\
\hline BIPS-606 & 7135 & $4 / 4$ & 30 \\
BIPS-1998 & 15066 & $4 / 4$ & 70 \\
BIPS-2476 & 16861 & $4 / 4$ & 100 \\
BIPS-3078 & 21128 & $4 / 4$ & 120 \\
\hline
\end{tabular}


The models are named on the number of the states they consist of [24]. Detailed of those models are available on the web-page.

In the previous work [12], it is found that IRKA based reduced-order model for the semi-stable model BIPS-3078 has no finite solution of the corresponding Riccati equation but the ISKA approach has no such kind of limitation.

\subsection{Frequency domain analysis}

All the above-mentioned power system models are structurally identical and have the same physical attributes. For the compactness of the work, only the comparative analysis of the transfer function of model BIPS-3078 is provided.

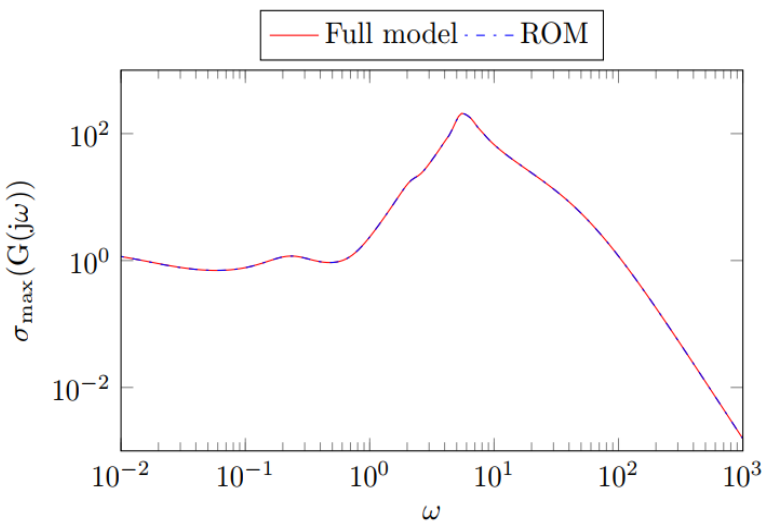

(a) Sigma plot

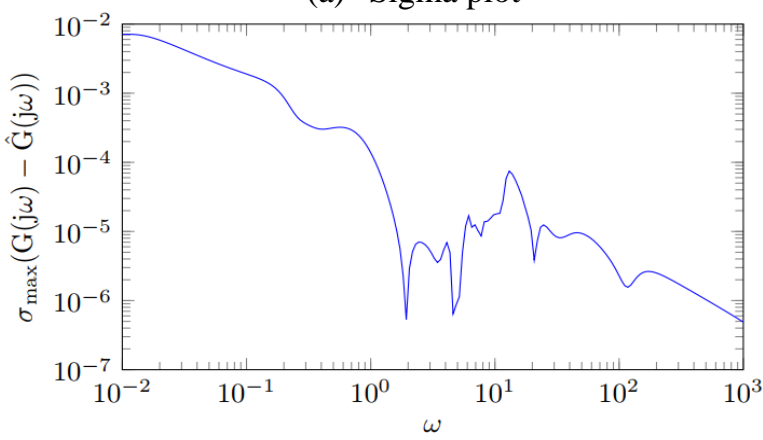

(b) Absolute error

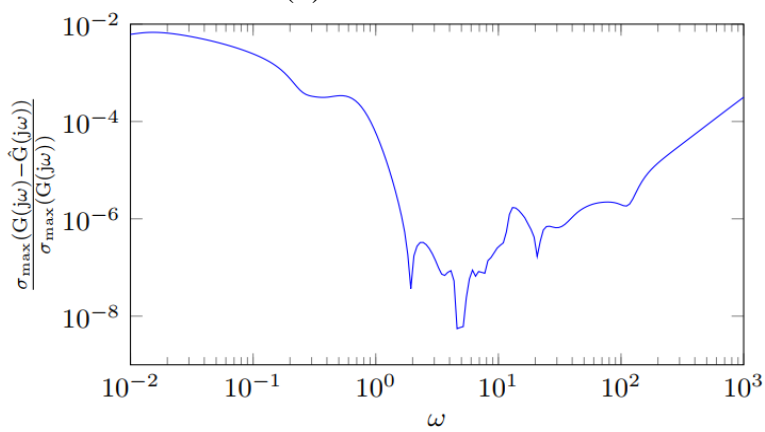

(c) Relative error

Fig. 1 Comparison of full model and ROM of the model BIPS3078

Sub-figures of Fig. 1 gives graphical validation of the efficient match of the transfer function of model BIPS-3078 with the corresponding ISKA-based ROM. Fig. 1a displays comparison transfer functions, whereas Fig. 1b and Fig. 1c depict the absolute error and relative error in computing the ROM of the target model.

From the above-displaying figures, it can be said that the ISKA-based approach is efficient for finding desired ROM of the target model.

\subsection{Stability analysis}

Sub-figures of Fig. 2 exhibit the stabilized step-responses of the model BIPS-3078 for the dominant input-output relations.

From the foregoing figures, it has been seen that in every input-output relation, the step-responses of the model BIPS-3078 optimally stabilized.

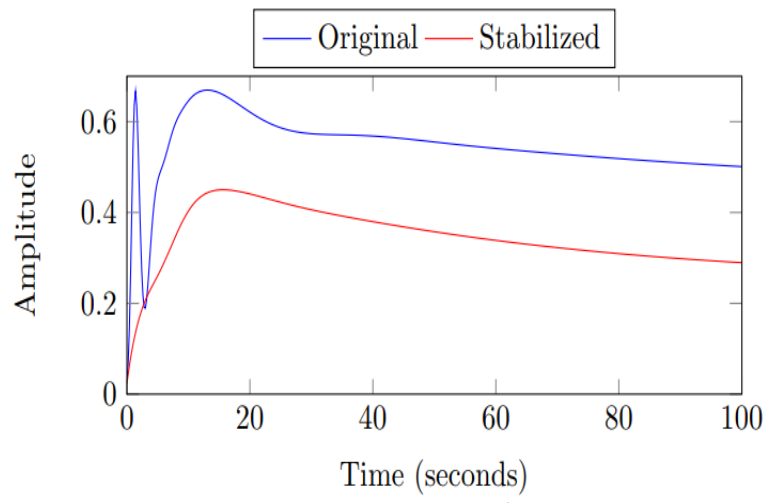

(a) $1^{\text {st }}$ input $/ 4^{\text {th }}$ input

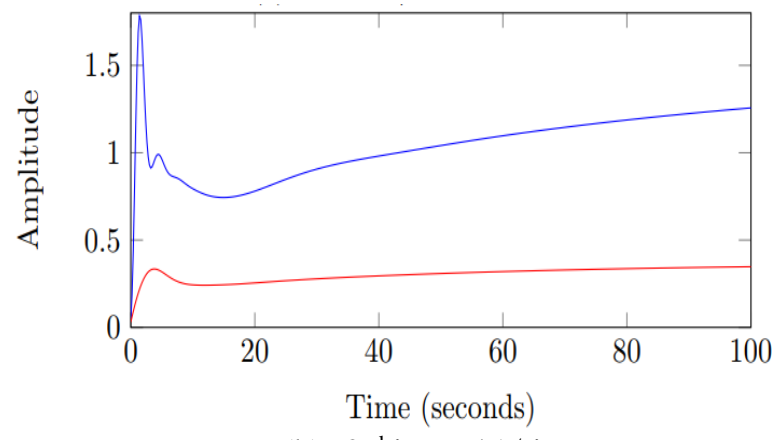

(b) $2^{\text {nd }}$ input $/ 1^{\text {st }}$ input

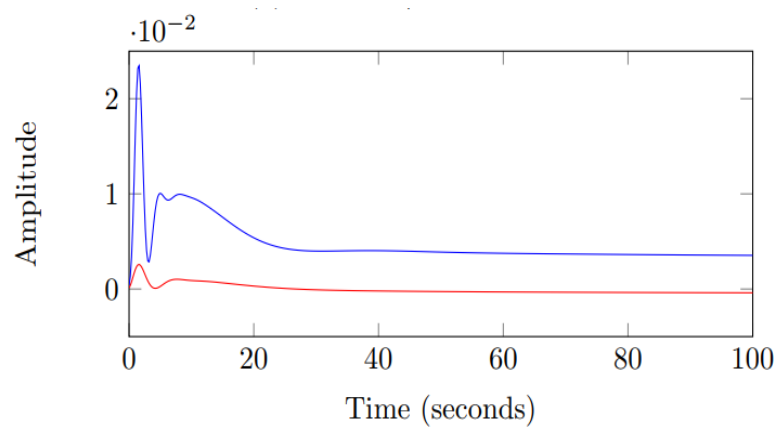

(c) $3^{\text {rd }}$ input $/ 2^{\text {nd }}$ input

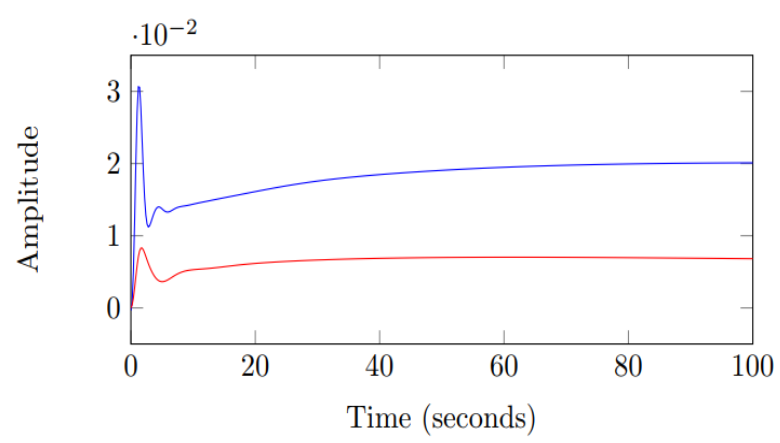

(d) $4^{\text {th }}$ input $/ 3^{\text {rd }}$ input

Fig. 2 Stabilization of step-responses of the model BIPS-3078

From the aforesaid figures, it is revealed that the eigenvalues of the models BIPS-606, BIPS-1998, and BIPS-2476 are stabilized efficiently. 
Sub-figures of Fig. 3 demonstrate the optimal feedback stabilization of the eigenvalues of the BIPS models.

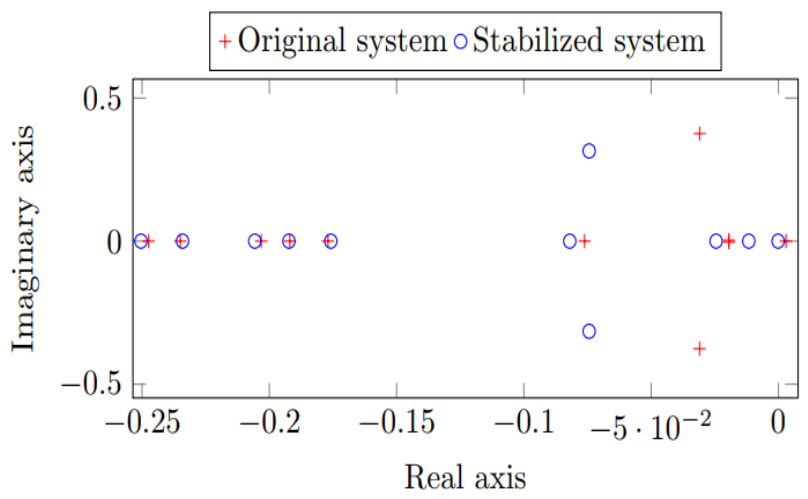

(a) BIPS-606

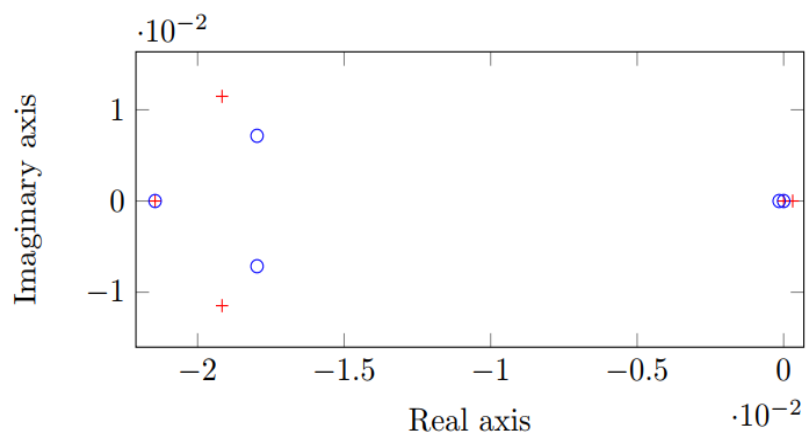

(b) BIPS-1998

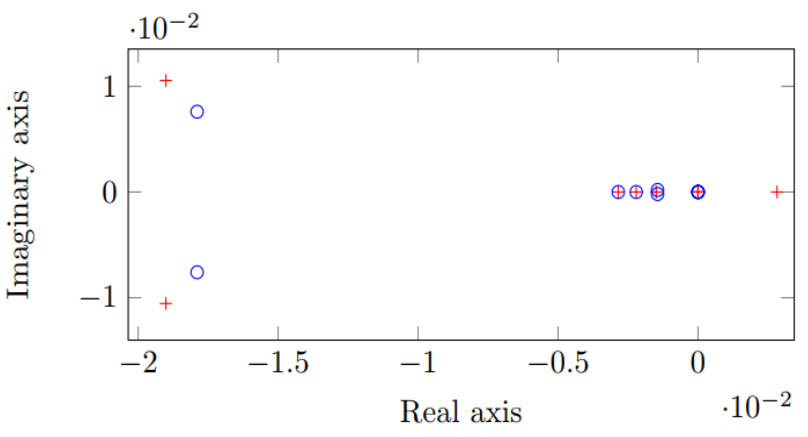

(c) BIPS-2476

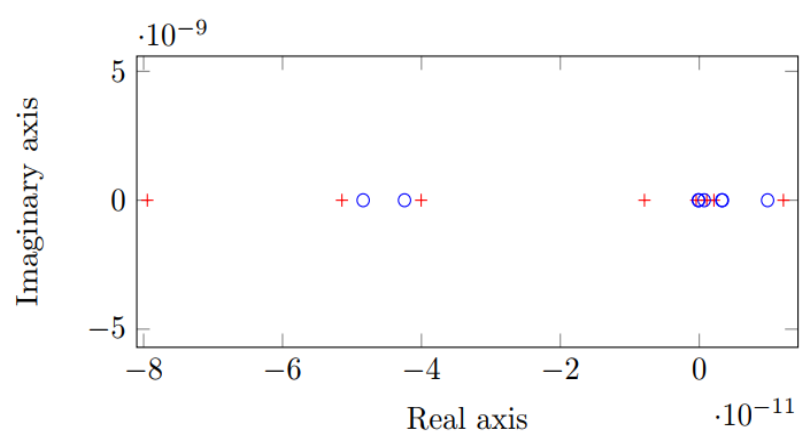

(d) BIPS-3078

Fig. 3 Eigenvalue stabilization of BIPS models

But the stabilization process for the semi-stable model BIPS-3078 is mildly interrupted but still acceptable as the eigenvalues are in the very small neighborhood of the imaginary axis (a very magnified view is provided).

\section{$4.3 \boldsymbol{H}_{\mathbf{2}}$-norm comparisons}

Table 2 represents the $\mathcal{H}_{2}$ error norm of the full model and the corresponding ROM of the BIPS models. This table is numerical evidence of the robustness of the proposed approach.

Table $2 \mathcal{H}_{2}$ error norm the of the full models and the ROMs

\begin{tabular}{|c|c|c|c|c|}
\hline Model & $\begin{array}{c}\text { BIPS- } \\
\mathbf{6 0 6}\end{array}$ & $\begin{array}{c}\text { BIPS- } \\
\mathbf{1 9 9 8}\end{array}$ & $\begin{array}{c}\text { BIPS- } \\
\mathbf{2 4 7 6}\end{array}$ & $\begin{array}{c}\text { BIPS- } \\
\mathbf{3 0 7 8}\end{array}$ \\
\hline $\boldsymbol{H}_{\mathbf{2}}$ & 2.16 & 1.22 & 4.34 & 2.05 \\
error & $\times 10^{0}$ & $\times 10^{-2}$ & $\times 10^{-4}$ & $\times 10^{-4}$ \\
norm & & & & \\
\hline
\end{tabular}

It has been audited that the $\mathcal{H}_{2}$ error norms are decreasing with the increasing size of the target models. Thus, the ISKA approach is expedient to minimize the $\mathcal{H}_{2}$ error norms of the considering models and it gives a better approximation for the models of the larger size.

\section{Conclusion}

We have discussed a sparsity-preserving two-sided projection-based reduced-order modelling approach for index1 descriptor systems of the first-order form. The Gramian based singular value decomposition and Krylov-based reduced-order modelling are coupled to achieve the reduced-order models. The conventional first-order LRCF-ADI and ISKA algorithms are modified to the sparse form keeping the structure of the target systems invariant. The proposed techniques are devoted to the Riccati-based feedback stabilization of the target systems. We have tested the validity of the proficiency of the derived approaches by implementing them to the power system models of the type unstable index-1 descriptor system in the first-order form. The robustness is investigated by $\mathcal{H}_{2}$ error norms of the reduced-order models corresponding to the target models.

\section{Acknowledgment}

The work is supported by Institute for Advanced Research (IAR), United International University, Dhaka, Bangladesh. The project title is "Computation of Optimal Control for Differential-Algebraic Equations (DAE) with Engineering Applications" with the reference IAR/01/19/SE/18.

\section{References}

[1] Hossain, M.S. and Uddin, M.M., 2019. Iterative methods for solving large sparse Lyapunov equations and application to model reduction of index 1 differentialalgebraic equations. Numerical Algebra, Control \& Optimization, 9(2), p.173.

[2] Benner, P., Saak, J. and Uddin, M.M., 2016, December. Reduced-order modeling of index-1 vibrational systems using interpolatory projections. In 2016 19th International Conference on Computer and Information Technology (ICCIT) (pp.134-138). IEEE.

[3] Uddin, M.M., 2015. Computational methods for model reduction of large-scale sparse structured descriptor systems (Doctoral dissertation, Otto-von Guericke Universität Magdeburg).

[4] Benner, P., Saak, J. and Uddin, M.M., 2016. Structure preserving model order reduction of large sparse secondorder index-1 systems and application to a mechatronics 
model. Mathematical and Computer Modelling of Dynamical Systems, 22(6), pp.509-523.

[5] Rahman, M., Uddin, M.M., Andallah, L.S. and Uddin, M., 2020. Interpolatory Projection Techniques for $\mathcal{H}_{2}$ Optimal Structure-Preserving Model Order Reduction of Second-Order Systems. Advances in Science, Technology and Engineering Systems Journal, 5(4), pp.715-723.

[6] Chu, E.K.W., 2011, August. Solving large-scale algebraic Riccati equations by doubling. In Talk presented at the Seventeenth Conference of the International Linear Algebra Society, Braunschweig, Germany (Vol. 22).

[7] Chen, W. and Qiu, L., 2015. Linear quadratic optimal control of continuous-time LTI systems with random input gains. IEEE Transactions on Automatic Control, 61(7), pp.2008-2013.

[8] Abou-Kandil, H., Freiling, G., Ionescu, V. and Jank, G., 2012. Matrix Riccati equations in control and systems theory. Birkhäuser.

[9] Bänsch, E., Benner, P., Saak, J. and Weichelt, H.K., 2015. Riccati-based boundary feedback stabilization of incompressible Navier-Stokes flows. SIAM Journal on Scientific Computing, 37(2), pp.A832-A858.

[10] Uddin, M., Khan, M.A.H. and Uddin, M.M., 2019, September. Riccati based optimal control for linear quadratic regulator problems. In 2019 5th International Conference on Advances in Electrical Engineering (ICAEE) (pp.290-295). IEEE.

[11] Uddin, M., Khan, M.A.H. and Uddin, M.M., 2019, December. Efficient computation of Riccati-based optimal control for power system models. In 2019 22nd International Conference on Computer and Information Technology (ICCIT) (pp.260-265). IEEE.

[12] Uddin, M., Uddin, M.M., Khan, M.A.H. and Rahman, M.M., 2021. Interpolatory projection technique for Riccati-based feedback stabilization of index-1 descriptor systems. IOP Conference Series: Materials Science and Engineering, 1070(1), pp.12-22.

[13] Li, J.R., 2000. Model reduction of large linear systems via low rank system Gramians (Doctoral dissertation, Massachusetts Institute of Technology).

[14] Hossain, M.S., Omar, S.G., Tahsin, A. and Khan, E.H., 2017, September. Efficient system reduction modeling of periodic control systems with application to circuit problems. In 2017 4th International Conference on
Advances in Electrical Engineering (ICAEE) (pp. 259264). IEEE.

[15] Gugercin, S., Antoulas, A.C. and Beattie, C., 2008. $\mathrm{H}_{2}$ model reduction for large-scale linear dynamical systems. SIAM journal on matrix analysis and applications, 30(2), pp.609-638.

[16] Wyatt, S.A., 2012. Issues in interpolatory model reduction: Inexact solves, second-order systems and DAEs (Doctoral dissertation, Virginia Tech).

[17] Gugercin, S., 2008. An iterative SVD-Krylov based method for model reduction of large-scale dynamical systems. Linear Algebra and its Applications, 428(8-9), pp.1964-1986.

[18] Khatibi, M., Zargarzadeh, H. and Barzegaran, M., 2016, September. Power system dynamic model reduction by means of an iterative SVD-Krylov model reduction method. In 2016 IEEE Power \& Energy Society Innovative Smart Grid Technologies Conference (ISGT) (pp.1-6). IEEE.

[19] Li, S., Trevelyan, J., Wu, Z., Lian, H., Wang, D. and Zhang, W., 2019. An adaptive SVD-Krylov reduced order model for surrogate based structural shape optimization through isogeometric boundary element method. Computer Methods in Applied Mechanics and Engineering, 349, pp.312-338.

[20] Lu, A. and Wachspress, E.L., 1991. Solution of Lyapunov equations by alternating direction implicit iteration. Computers \& Mathematics with Applications, 21(9), pp.43-58.

[21] Benner, P., Li, J.R. and Penzl, T., 2008. Numerical solution of large-scale Lyapunov equations, Riccati equations, and linear-quadratic optimal control problems. Numerical Linear Algebra with Applications, 15(9), pp.755-777.

[22] Benner, P., Køhler, M. and Saak, J., 2011. Sparse-dense Sylvester equations in $\mathcal{H}_{2}$ model order reduction.

[23] Freitas, F.D. and Costa, A.S., 1999. Computationally efficient optimal control methods applied to power systems. IEEE transactions on power systems, 14(3), pp.1036-1045.

[24] Leandro, R.B., e Silva, A.S., Decker, I.C. and Agostini, M.N., 2015. Identification of the oscillation modes of a large power system using ambient data. Journal of Control, Automation and Electrical Systems, 26(4), pp.441-453. 